\section{Endocytosis by macrophages: interplay of macrophage scavenger receptor-1 and LDL receptor-related protein-1}

Multiple receptors may mediate the cellular uptake of a single protein and thereby affect the plasma level of the involved protein. In case of Von Willebrand factor (VWF) these receptors include LDL receptor-related protein-1 (LRP-1), Macrophage scavenger receptor-1 (MSR-1, SRAI or CD204), the Macrophage Galactose-type lectin
(CLEC10A, MGL or CD301), Siglec-5 and the Asialoglycoprotein receptor (ASGPR). ${ }^{1}$ In the present study, we aimed to gain insight into the interplay of multiple receptors to the cellular internalization of a single ligand like VWF.

The macrophages in the liver and spleen have been reported to contribute considerably to the cellular uptake of VWF. ${ }^{2-4}$ Previously, we have shown that also human monocyte-derived macrophages (MDM) internalize VWF via a mechanism that depends on LRP $-1 .{ }^{5}$ We now analyzed the cell surface proteome of $\mathrm{MDM}$ using mass

A Mature VWF \begin{tabular}{|l|l|l|l|l|l|l|l|l|l|l|l|l|l|}
\hline$D^{\prime}$ & $\mathrm{A} 3$ & $\mathrm{~A} 1$ & $\mathrm{~A} 2$ & $\mathrm{~A} 3$ & $\mathrm{D} 4 \mathrm{~N}$ & $\mathrm{D} 4$ & $\mathrm{C} 1$ & $\mathrm{C} 2$ & $\mathrm{C} 3$ & $\mathrm{C} 4$ & $\mathrm{C} 5$ & $\mathrm{C} 6$ & $\mathrm{CK}$ \\
\hline
\end{tabular} VWF Fragments D' $\mathrm{D} 3 \mathrm{HPC4}$ D' $\mathrm{D} 3 \mathrm{~A}-\mathrm{HPC4}$

B

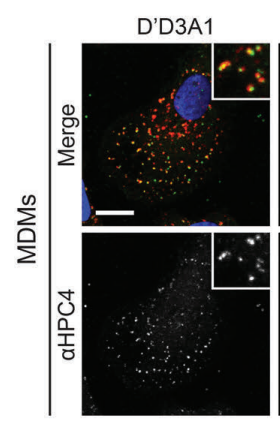

D

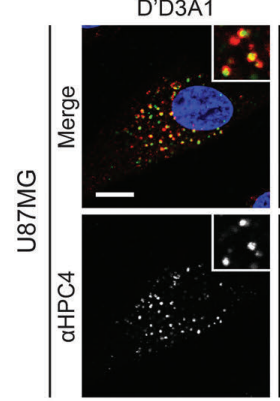

$\mathbf{F}$
D'D3

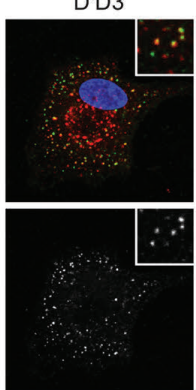

D'D3

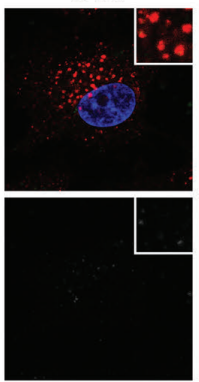

$+5 \times$ RAP

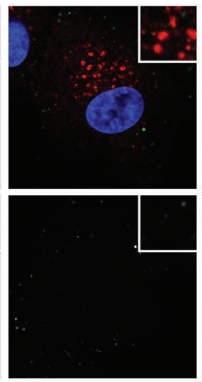

No Fragment

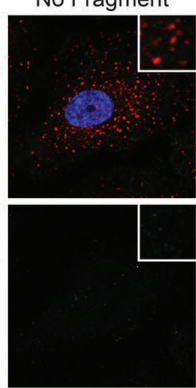

No Fragment

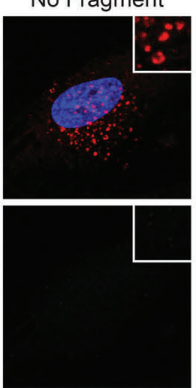

$+5 \times$ Cluster-II

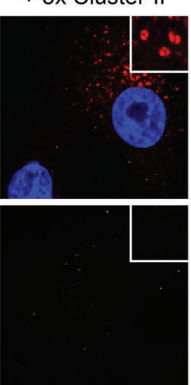

C

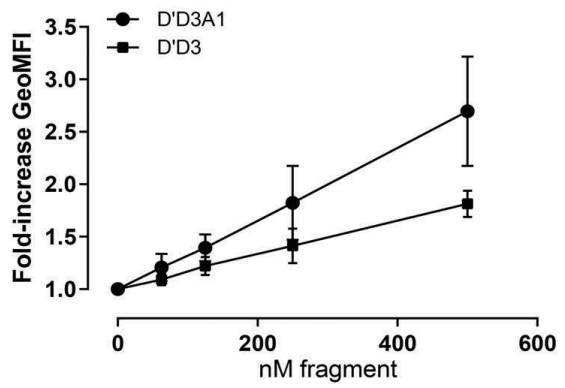

E

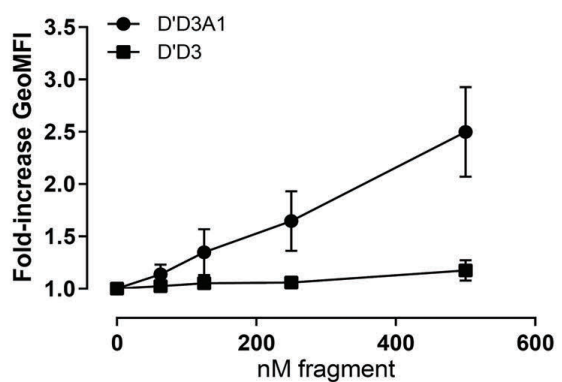

G

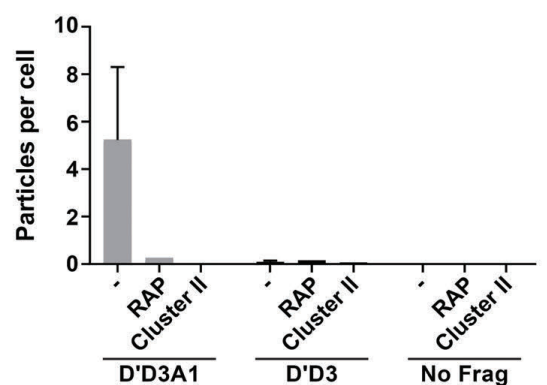

Figure 1. Uptake of VWF fragments D'D3A1 and D'D3 in MDMs and U87MG cells. (A) Schematic representation of the domains of VWF and the generated fragments. Both fragments were equipped with a C-terminal HPC4 tag for purification and detection purposes. (B,D) Uptake of 100 nM D'D3A1 or D'D3 in MDM and U87MG cells. Cells were stained with antibodies against HPC4 (green) and EEA1 (red), and Hoechst (blue). Scale bar represents 10 um. Sites of co-localization result in a yellow staining. (C,E) Flow cytometry measurements of fragment uptake in MDM (C) and U87MG cells (E). Cells were incubated with concentrations of fragments of $0,63,125,250$ and $500 \mathrm{nM}$. Data show the fold-increase (mean \pm standard deviation [SD]) of the geometric mean fluorescent intensity (GeoMFI) after correction for background staining in cells incubated with buffer only. Data were obtained from four independent experiments. (F) U87MG cells were incubated with 75 nM D'D3A1 or D'D3 with or without a five times molar excess of RAP or LRP-1 Cluster-II. Cells were stained with anti-HPC4 (green), antiEEA1 (red) and Hoechst (blue). Scale bar represents $10 \mu \mathrm{m}$. Sites of co-localization result in a yellow staining (G) Quantification of confocal images. Number of particles per cell were counted in tile scans from four independent experiments. For each experiment 300-500 cells were analyzed. Data represent mean \pm SD. 
spectrometry analysis to identify putative other VWF clearance receptors on MDM. To this end, extracellular proteins were labelled with membrane impermeable sulfo-NHS-biotin after which samples were subjected to mass spectrometry processing and enrichment of biotinlabeled peptides using a biotin pull-down approach. Mass spectrometry analysis of the collected proteins resulted in the identification of more than a thousand potential cell surface proteins (Online Supplementary Figure S1). Several peptides of the VWF receptors LRP-1 and MSR-1 were identified as well as one peptide that is shared by Siglec5 and Siglec-14. The estimated copy numbers of these receptors revealed a markedly higher expression of LRP1 and MSR-1 compared to Siglec-5/14. We therefore

A
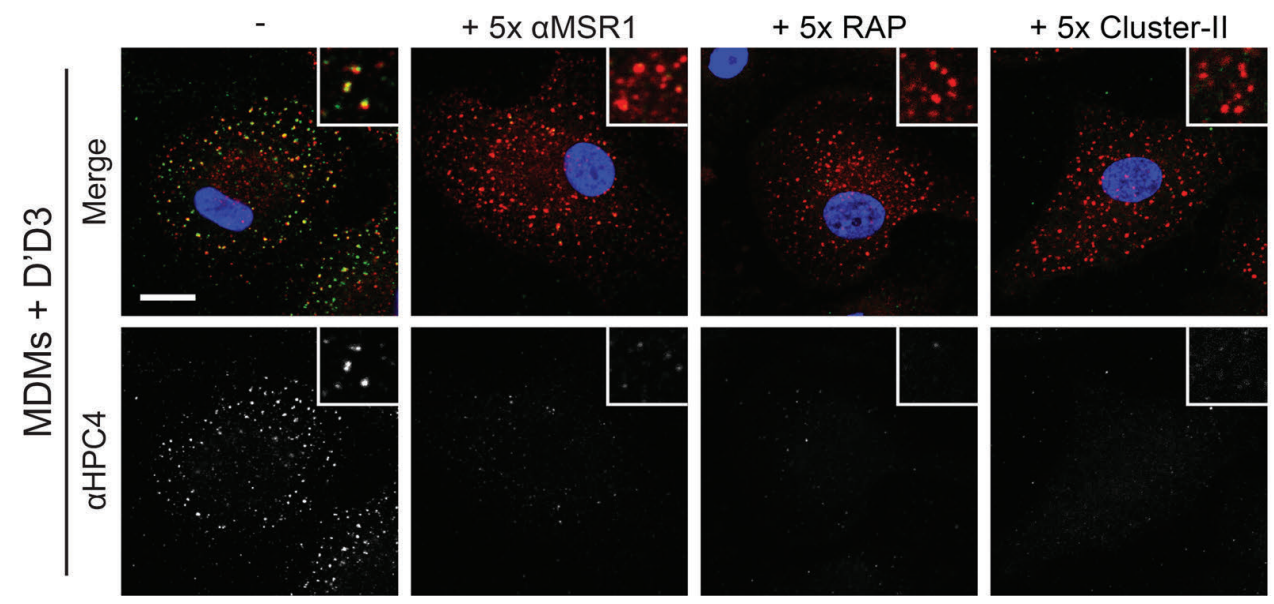

B
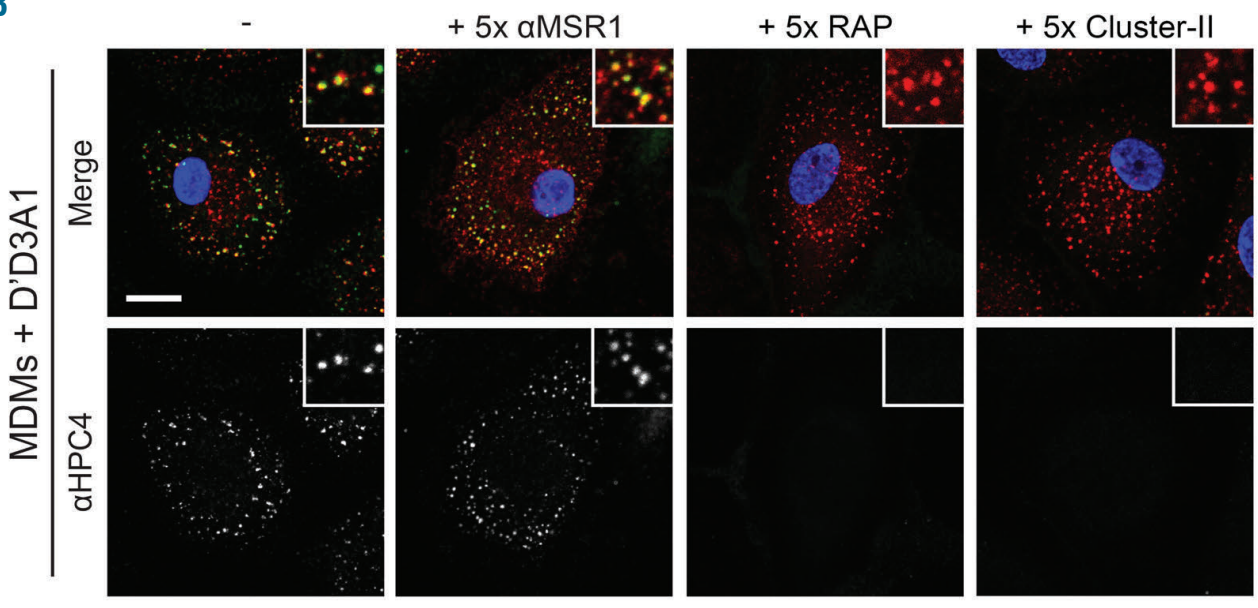

C

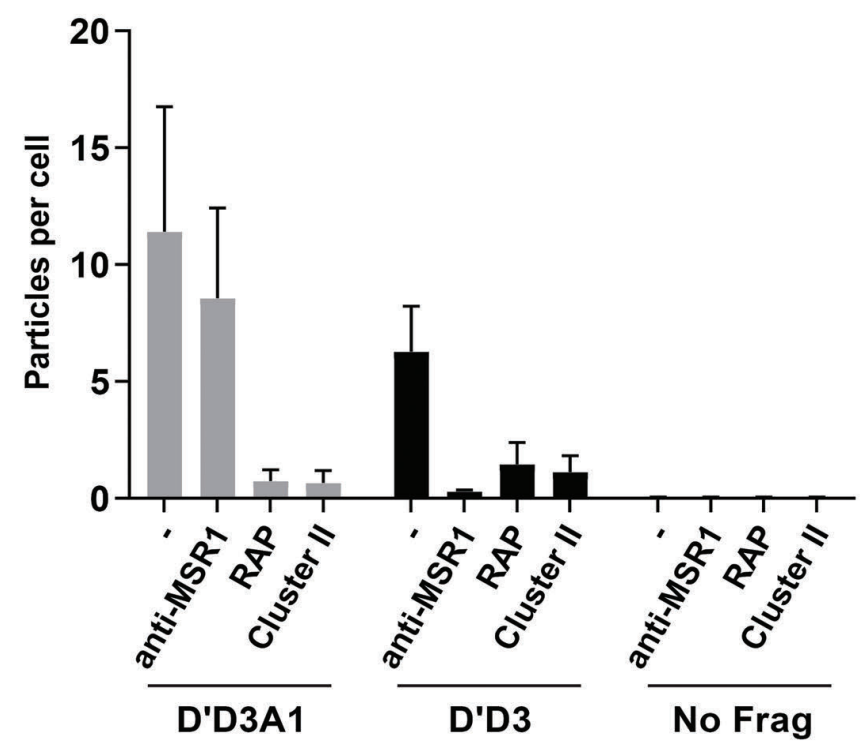

Figure 2. Uptake of D'D3 by MDM is mediated by MSR1 and LRP-1. (A,B) Blocking MSR-1 only affects D'D3 interaction, whereas blocking LRP-1 affects both fragments. MDM were incubated with $75 \mathrm{nM}$ D'D3A1 or D'D3 with or without a five times molar excess of antiMSR1 blocking antibodies, RAP or LRP-1 Cluster-II. Cells were stained with anti-HPC4 (green), anti-EEA1 (red) and Hoechst (blue). Sites of co-localization result in yellow staining. In MSR-1 blocking conditions, red staining represents EEA-1 as well as MSR-1 as these antibodies were from the same species and isotype. Scale bar represents $10 \mu \mathrm{m}$. (C) Quantification of confocal images. Number of particles per cell were counted in tile scans from four independent experiments. For each experiment 300-500 cells were analyzed. Data in C represents mean \pm SD. 
focused our study on the possible dual mechanism by which LRP-1 and MSR-1 may cooperate in the cellular uptake of VWF by MDM.

Studying the uptake mechanism of VWF is hampered by the fact that flow-induced shear force is required to facilitate VWF internalization by MDM. For LRP-1, others have previously shown that full-length VWF only binds LRP-1 under shear stress conditions. ${ }^{6}$ This observa- tion is compatible with our previous finding the MDM internalize VWF via LRP-1 in a flow-dependent manner. ${ }^{5}$ Shear force may induce a configurational change in VWF from a globular shape to an elongated shape, thereby exposing binding sites for clearance receptors like LRP-1 and possibly also MSR-1. In the present study, confocal microscopy and flow cytometry analysis showed that $\mathrm{N}$ terminal VWF fragments, comprising the D'D3 and

A

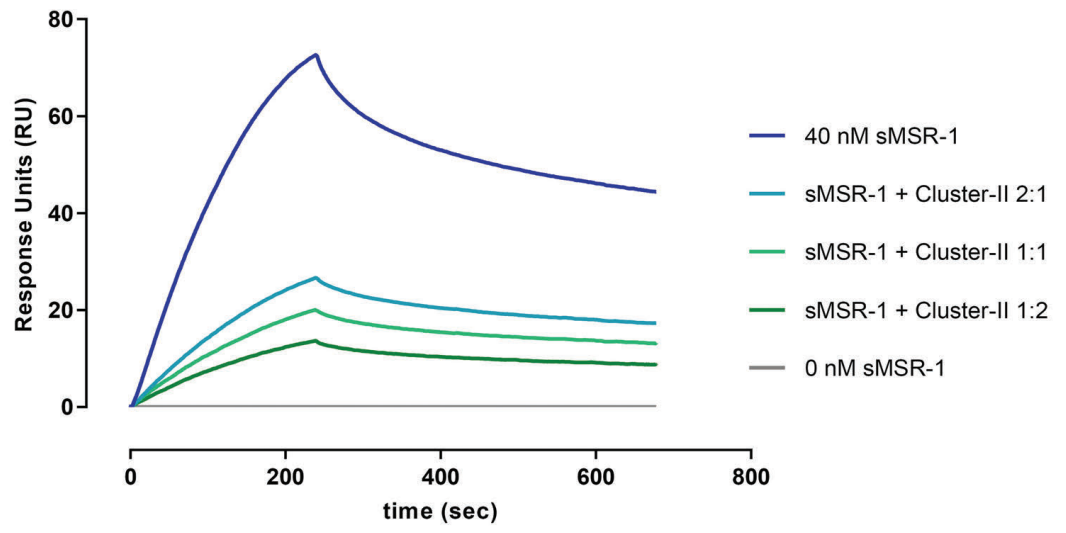

B U87MG + SMSR-1 $+5 x$ Cluster-II
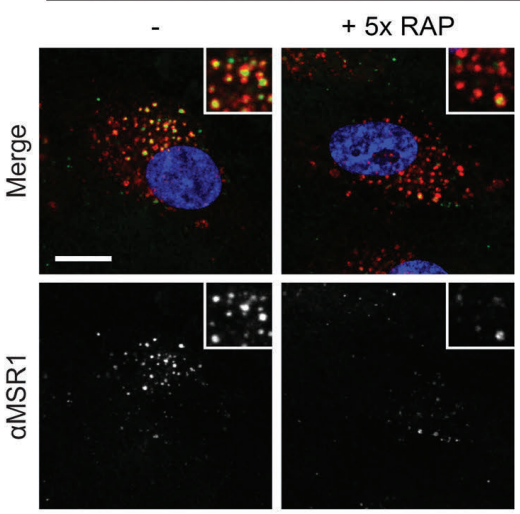

C
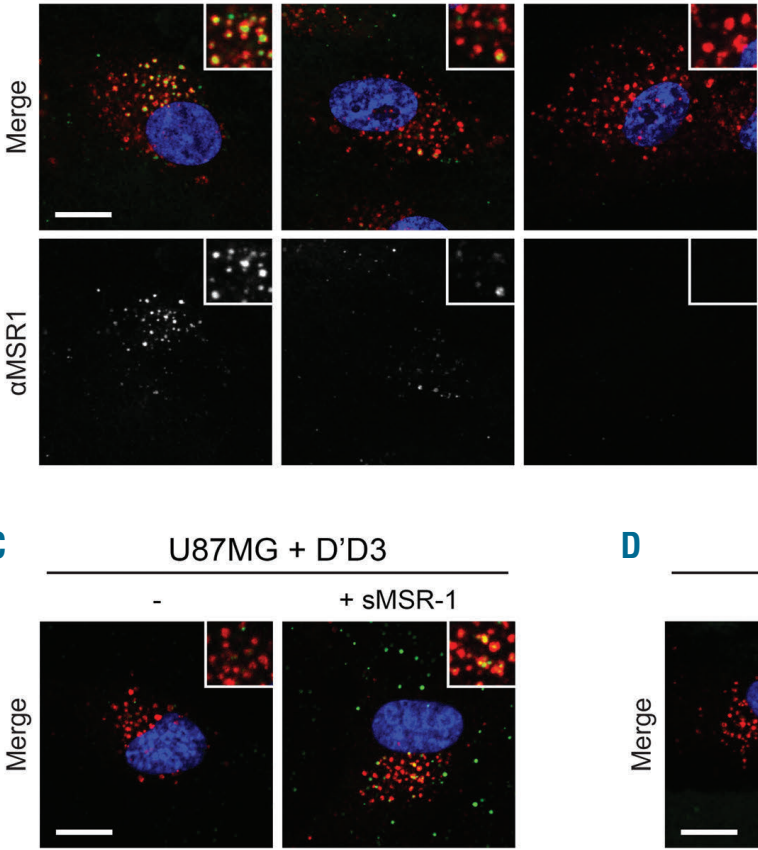

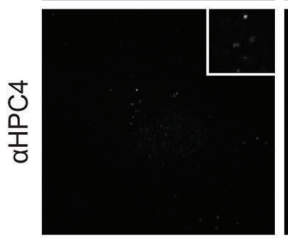

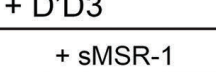
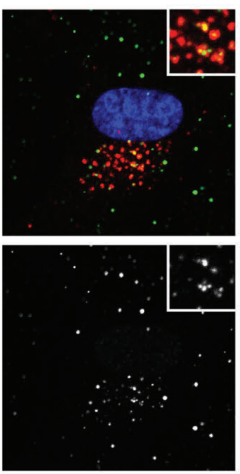

D

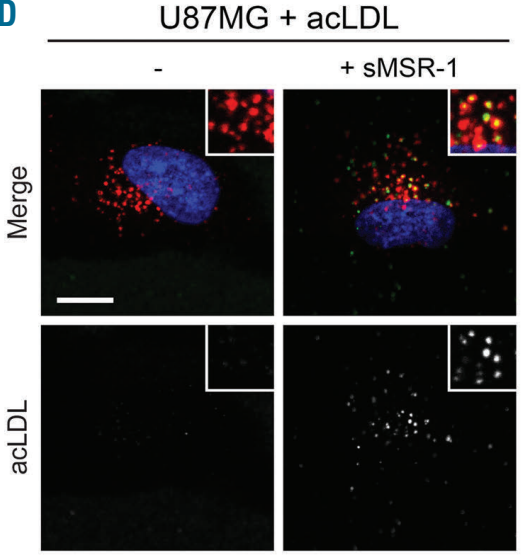

Figure 3. MSR-1 associates with LRP-1 to mediate D'D3 uptake. SPR and cell-based experiments to explore the interaction between MSR-1 and LRP-1. SPR and cell-based experiments to explore the interaction between MSR-1 and LRP-1. (A) Binding of soluble MSR-1 (sMSR-1) to 18 fmol/mm2 immobilized LRP-1 Cluster-II in the absence and presence of increasing concentrations of soluble Cluster-II. The concentration of sMSR- 1 was kept at 40 nM and the concentration of soluble Cluster-II was 20, 40 or 80 nM. (B) Uptake of $100 \mathrm{nM}$ sMSR-1 by U87MG cells in the absence or presence of a five times molar excess of RAP or Cluster-II. Cells were stained with anti-MSR-1 (green), anti-EEA1 (red) and Hoechst (blue). Sites of co-localization result in yellow staining. (C) Uptake of 100 nM D'D3 in the absence and presence of $200 \mathrm{nM}$ sMSR-1. Cells were stained for anti-HPC4 (green), anti-EEA1 (red) and Hoechst (blue). (D) Uptake of $2.5 \mu \mathrm{g} / \mathrm{mL}$ acLDL in the absence and presence of $200 \mathrm{nM}$ sMSR-1. The scale bar represents $10 \mu \mathrm{m}$. 
D'D3A1 domains, were effectively internalized by MDM in a shear-independent manner (Figure 1A-C). This observation may fit the model that the receptor binding sites of VWF are accessible for interaction with LRP-1 and/or MSR-1 within the D'D3A1 fragment.

To further dissect the role of LRP-1 in these observations, we utilized the U87MG cell line, which has been shown to express relatively high levels of LRP-1. ' Our mass spectrometry approach confirmed the expression of LRP-1 in these cells and showed that MDM and U87MG cells contained similar levels of LRP-1 (Online Supplementary Table S1). Although we cannot fully exclude their presence using this approach, MSR-1 and other VWF clearance receptors were not identified on U87MG cells (Online Supplementary Table S1). Microscopy and flow cytometry studies revealed that U87MG cells effectively internalized D'D3A1, but not D'D3 (Figure 1D-E). The presence of LRP-antagonist Receptor Associated Protein (RAP) abrogated the internalization of D'D3A1 by these cells (Figure 1F-G). Likewise, the cellular uptake of D'D3A1 was blocked in the presence of an excess of purified LRP-1 domain Cluster II. These findings indicate that LRP-1 mediates the uptake of D'D3A1 by U87MG cells. These observations are in line with the finding that the A1 domain of VWF comprises a binding site for LRP-1. ${ }^{8}$ Indeed, surface plasmon resonance (SPR) analysis showed that, in contrast to D'D3, D'D3A1 did bind to Cluster II, thereby confirming that the A1 domain of D'D3A1 is crucial for the interaction with LRP-1 (Online Supplementary Table S2).

In parallel, we investigated the role of MSR-1 in the internalization of VWF fragments D'D3 and D'D3A1. Binding studies showed a dose-dependent interaction of the extracellular domain of MSR-1 (sMSR-1) with both VWF fragments (Online Supplementary Figure S3). This observation is in agreement with the study by Wohner et al. that revealed binding sites for MSR-1 in the D'D3 and the A1 domain of VWF. ${ }^{9}$ However, internalization experiments revealed that only the cellular uptake of D'D3 can be blocked by an antibody directed against MSR-1 (Figure 2). As the antibody inhibits both the binding of D'D3 and D'D3A1 to the ligand binding domain of MSR1 (Online Supplementary Figure S4), this indicates that the uptake of D'D3A1 proceeds via an alternative mechanism, possibly via LRP-1. To address this issue, MDM were incubated with the fragments in the presence of the LRP antagonist RAP or an excess Cluster II. Competing with RAP or Cluster II minimized the uptake of the D'D3A1 fragment in MDM (Figure 2B). Surprisingly, the uptake of D'D3 by MDM was similarly affected (Figure 2A). This suggests that internalization of both D'D3 and D'D3A1 is dependent on LRP-1 in spite of the notion that the LRP-1 binding site resides in the A1 domain of VWF.

To explain the remarkable dependence of D'D3 uptake by MDM on both LRP-1 and MSR-1, we hypothesized that MSR-1-induced D'D3 internalization may be mediated by LRP-1. We therefore assessed whether SMSR-1 can directly interact with LRP-1 using SPR analysis. To this end, sMSR-1 was passed over immobilized Cluster II on an SPR sensor chip. A dose dependent-binding response was observed that could be effectively blocked by prior incubation of sMSR-1 with Cluster II (Figure 3A). This finding implies that sMSR-1 may indeed interact with LRP-1. Subsequent confocal microscopy analysis showed that, upon incubation with U87MG cells, sMSR1 was transported to the early endosomes via a process that was inhibited in presence of an excess of RAP or Cluster II (Figure 3B). These observations imply that sMSR-1 is endocytosed by U87MG cells via an LRP-1 dependent mechanism. In line with this hypothesis, we found that sMSR-1 could induce the internalization of D'D3 by U87MG cells (Figure 3C). Taken together, these data suggest that sMSR-1 may act as a bridging receptor between LRP-1 and D'D3, thereby mediating the uptake of this fragment via LRP-1. To assess whether this mechanism of endocytosis also holds true for another MSR-1 ligand, we incubated fluorescent acLDL with the U87MG cells in the presence and absence of sMSR-1. No uptake of acLDL was observed in the absence of sMSR-1, however, as for D'D3, the presence of sMSR-1 induced localization of acLDL to the early endosomes (Figure 3D).

These data provide evidence for a role for LRP-1 as a co-receptor for MSR-1 in the uptake of ligands by MDM. A similar mechanism has been proposed for the uptake of the uPA-PAI-1 complex via the urokinase plasminogen activator receptor (uPAR), although details are different. ${ }^{10}$ The UPAR can bind urokinase plasminogen activator (UPA) with and without its inhibitor PAI-1. The UPARuPA complex is stable, whereas the uPAR-uPA-PAI-1 complex is internalized via LRP- $1 .{ }^{10}$ Also in this case, LRP1 serves as a co-receptor which, in concert with a primary receptor (uPAR), mediates the internalization of a ligand (uPA-PAI-1). We and others have previously shown that the LRP-1 dependent uptake of Factor VIII (FVIII) also proceeds via a dual receptor mechanism. In addition to the interaction with LRP-1, FVIII requires prior binding to other structural elements on the cell surface to mediate the internalization by the cells. ${ }^{11,12}$ These findings indicate that LRP-1 may serve as a co-receptor for multiple primary receptors, and multiple ligands.

Studies in mice have shown that LRP-1 and MSR-1 play a physiological role in VWF clearance as well. ${ }^{6,9}$ However, many issues involving this mechanism remain to be resolved. For instance, others have shown that PMA-stimulated THP-1 cells can bind VWF and internalize it via a shear-independent mechanism., ${ }^{9,13}$ In our hands, MDM only internalize VWF in a shear-dependent manner. Understanding the differences between these cellular systems remains a topic for further investigation. The same holds true for the expanding list of putative endocytic receptors for VWF. The relative importance of these receptors to mediate the uptake of VWF remains an open question.

Based on the data presented in this study, we propose the following model to explain the relationship between the two abundant VWF receptors on MDM. Both LRP-1 and MSR-1 associate with regions within D'D3A1, thereby initiating two endocytic pathways that are both regulated by LRP-1. The first pathway follows a direct association of the VWF A1 domain to LRP-1. In the second pathway, VWF interacts to MSR-1 via regions in the D'D3 assembly, which subsequently associates to LRP-1 for endocytosis.

Eelke P. Béguin,' Małgorzata A. Przeradzka,' Esmée FJ. Janssen, ${ }^{1}$ Henriët Meems, ${ }^{1}$ Magdalena Sedek, ${ }^{1}$ Carmen van der Zwaan, ${ }^{1}$ Koen Mertens, ${ }^{1,2}$ Maartje van den Biggelaar, ${ }^{1,3}$ Alexander B. Meijer ${ }^{1,3}$ and Marjon J. Mourik

${ }^{1}$ Department of Molecular and Cellular Hemostasis, Sanquin Research, Amsterdam; 'Department of Pharmaceutical Sciences, Utrecht Institute for Pharmaceutical Sciences, Utrecht University, Utrecht; ${ }^{3}$ Department of Biomolecular Mass Spectrometry and Proteomics, Utrecht Institute for Pharmaceutical Sciences, Utrecht University, Utrecht, the Netherlands

\section{Correspondence: ALEXANDER B. MEIJER}

s.meijer@sanquin.nl

doi:10.3324/haematol.2018.210682 
Information on authorship, contributions, and financial \& other disclosures was provided by the authors and is available with the online version of this article at WWw. haematologica.org.

\section{References}

1. O'Sullivan JM, Ward S, Lavin M, O'Donnell JS. Von Willebrand factor clearance - biological mechanisms and clinical significance. Br J Haematol. 2018;183(2):185-195.

2. Rawley O, O'Sullivan JM, Chion A, et al. Von Willebrand factor arginine 1205 substitution results in accelerated macrophage-dependent clearance in vivo. J Thromb Haemost. 2015;13(5):821-826.

3. O'Sullivan JM, Aguila S, McRae E, et al. N-linked glycan truncation causes enhanced clearance of plasma-derived von Willebrand factor. J Thromb Haemost. 2016;14(12):2446-2457.

4. Van Schooten CJ, Shahbazi S, Groot E, et al. Macrophages contribute to the cellular uptake of Von Willebrand factor and factor VIII in vivo. Blood. 2008;112(5):1704-1712.

5. Castro-Núñez L, Dienava-Verdoold I, Herczenik E, Mertens K, Meijer $A B$. Shear stress is required for the endocytic uptake of the factor VIII-von Willebrand factor complex by macrophages. J Thromb Haemost. 2012;10(9):1929-1937.

6. Rastegarlari G, Pegon JN, Casari C, et al. Macrophage LRP1 contributes to the clearance of von Willebrand factor. Blood. 2012;119(9):2126-2134.
7. Maletínská L, Blakely EA, Bjornstad KA, Deen DF, Knoff LJ, Forte TM. Human glioblastoma cell lines: levels of low-density lipoprotein receptor and low-density lipoprotein receptor-related protein. Cancer Res. 2000;60(8):2300-2303.

8. Wohner N, Legendre P, Casari C, Christophe OD, Lenting PJ, Denis $\mathrm{CV}$. Shear stress-independent binding of von Willebrand factor-type 2B mutants p.R1306O \& p.V1316M to LRP1 explains their increased clearance. J Thromb Haemost. 2015;13(5):815-820.

9. Wohner N, Muczynski V, Mohamadi A, et al. Macrophage scavenger receptor SR-AI contributes to the clearance of von Willebrand factor. Haematologica. 2018;103(4):728-737.

10. Conese M. alpha-2 Macroglobulin receptor/Ldl receptor-related protein(Lrp)- dependent internalization of the urokinase receptor. J Cell Biol. 1995;131(6):1609-1622.

11. Sarafanov AG, Ananyeva NM, Shima M, Saenko EL. Cell Surface Heparan Sulfate Proteoglycans Participate in Factor VIII Catabolism Mediated by Low Density Lipoprotein Receptor-related Protein. J Biol Chem. 2001;276(15):11970-11979.

12. Castro-Núñez L, Koornneef JM, Rondaij MG, et al. Cellular uptake of coagulation factor VIII: Elusive role of the membrane-binding spikes in the C1 domain. Int J Biochem Cell Biol. 2017;89:34-41.

13. Chion A, O'Sullivan JM, Drakeford C, et al. N-linked glycans within the A2 domain of von Willebrand factor modulate macrophagemediated clearance. Blood. 2016;128(15):1959-1968. 